\title{
Recent results from proton intermittency analysis in nucleus-nucleus collisions from NA61 at CERN SPS
}

\author{
Nikolaos Davis* for the NA61/SHINE collaboration \\ Institute of Nuclear Physics, Polish Academy of Sciences \\ E-mail: nikolaos.daviseifj.edu.pl
}

\section{Nikolaos Antoniou}

University of Athens, Physics Department, Section of Nuclear and Elementary Particle Physics

E-mail: nantonio@phys.uoa.gr

\section{Fotios Diakonos}

University of Athens, Physics Department, Section of Nuclear and Elementary Particle Physics

E-mail: fdiakono@phys.uoa.gr

The search for experimental signatures of the critical point (CP) of strongly interacting matter [1] is one of the main objectives of the NA61/SHINE experiment at CERN SPS. In the course of the experiment, an energy (beam momentum $13 \mathrm{~A}-150 \mathrm{~A} \mathrm{GeV/c}$ ) and system size $(\mathrm{p}+\mathrm{p}, \mathrm{p}+\mathrm{Pb}$, $\mathrm{Be}+\mathrm{Be}, \mathrm{Ar}+\mathrm{Sc}, \mathrm{Xe}+\mathrm{La}$ ) scan is performed.

We investigate local proton density fluctuations connected to the critical behavior of the order parameter as a possible signature of the phase transition in the neighborhood of the CP. To this end, we perform an intermittency analysis of the proton second scaled factorial moments (SSFMs) in transverse momentum space, which we expect to scale according to a universal power-law in the vicinity of the $\mathrm{CP}$.

Previous analyses of this sort [2] revealed significant power-law fluctuations in the NA49 heavy ion collision experiment for the "Si"+Si system at $158 \mathrm{~A} \mathrm{GeV/c}$; no intermittency was observed in NA49 " $\mathrm{C}$ " $+\mathrm{C}$ and $\mathrm{Pb}+\mathrm{Pb}$ collisions at the same energy, nor in NA61/SHINE Be+Be collisions at $150 \mathrm{~A} \mathrm{GeV/c}$. The fitted power-law exponent in "Si"+Si was consistent with the theoretically expected critical value, within errors, a result suggesting a baryochemical potential for the critical point in the vicinity of $\sim 250 \mathrm{MeV}$. We now extend the analysis to the similar-sized NA61/SHINE $\mathrm{Ar}+\mathrm{Sc}$ system at $150 \mathrm{~A} \mathrm{GeV/c}$.

In the calculation of scaled factorial moments, statistical techniques are employed in order to subtract non-critical background and enhance the signal in cases of low statistics. Our analysis is supplemented by both critical and non-critical Monte Carlo simulations, through which we estimate non-critical background effects on the quality and magnitude of uncertainties of the intermittency power-law fit, as well as explore the possibility of non-critical effects producing an intermittency signal.

Corfu Summer Institute 2018 "School and Workshops on Elementary Particle Physics and Gravity" (CORFU2018)

31 August - 28 September, 2018

Corfu, Greece

${ }^{*}$ Speaker. 


\section{Introduction}

Experimental observables proposed for the detection of the QCD CP fall into two categories: event-by-event (global) fluctuations of integrated quantities [3, 4, 5], as well as local power-law fluctuations [6] of the order parameter of the QCD chiral phase transition, the chiral condensate $\langle\bar{q} q\rangle$. The critical properties of the chiral condensate are carried by the sigma field $\sigma(\mathbf{x})$, and may be probed indirectly through its decay into experimentally observable $\left(\pi^{+}, \pi^{-}\right)$pairs [7]. At finite baryochemical potential, critical fluctuations are also transferred to the net proton density, as well as to the proton and antiproton densities separately [8].

At the CP, the fluctuations of the order parameter are self-similar [9], belonging to the 3D-Ising universality class, and can be detected in transverse momentum space within the framework of an intermittency analysis of proton density fluctuations by use of scaled factorial moments (SFMs). A detailed analysis, augmented by properly adapted statistical techniques, can be found in Ref. [2], where we study various heavy nuclei collision datasets recorded in the NA49 experiment at maximum energy $\left(158 \mathrm{~A} \mathrm{GeV/c}, \sqrt{s_{N N}} \approx 17 \mathrm{GeV}\right)$ of the SPS (CERN).

\section{Method of analysis}

In a pure critical system, intermittency in transverse momentum space can be revealed by the scaling of the Second Scaled Factorial Moments (SSFMs) of protons as a function of bin size. For that purpose, a region of transverse momentum space is partitioned into a lattice of $M \times M$ equal-size bins. Consequently, the SSFMs:

$$
F_{2}(M)=\frac{\left\langle\frac{1}{M^{2}} \sum_{i=1}^{M^{2}} n_{i}\left(n_{i}-1\right)\right\rangle}{\left\langle\frac{1}{M^{2}} \sum_{i=1}^{M^{2}} n_{i}\right\rangle^{2}}
$$

are calculated, as an average over bins and events $(\langle\ldots\rangle)$, where $n_{i}$ is the number of particles in the $i$-th bin and $M^{2}$ is the total number of bins. If the system exhibits critical fluctuations, $F_{2}(M)$ is expected to scale with $M$, for large values of $M$, as a power-law:

$$
F_{2}(M) \sim M^{2 \phi_{2}}, \phi_{2}=\phi_{2, c r}^{B}=\frac{5}{6}
$$

where $\phi_{2}$ is the intermittency index, and provided the freeze-out occurs at exactly the critical point [10].

Noisy experimental data require the subtraction of a background of uncorrelated \& misidentified protons, which is achieved through the construction of correlation-free mixed events. A correlator can then be defined in terms of the moments of data and mixed events, as well as a mixed term,

$$
\Delta F_{2}(M)=F_{2}^{(d)}(M)-\lambda^{2}(M) F_{2}^{(m)}(M)-\lambda(M)(1-\lambda(M)) f_{b c} \quad ; \quad \lambda(M) \equiv \frac{\left\langle n_{b}\right\rangle}{\langle n\rangle}
$$


where $\lambda(M)$ is the ratio of background to data average particle multiplicities and $f_{b c}$ an unknown function. In the limiting case of a dominant background, $\lambda(M) \lesssim 1$, the critical behaviour is expected to be revealed in the approximate ${ }^{1}$ correlator estimate $(e)$,

$$
\Delta F_{2}^{(e)}(M) \simeq F_{2}^{(d)}(M)-F_{2}^{(m)}(M),
$$

where mixed event $(m)$ moments are simply subtracted from data $(d)$ moments. $\Delta F_{2}(M)$ should then scale as a power law, $\Delta F_{2}(M) \sim M^{2 \phi_{2}}$, in a limited range, with the same intermittency index as the pure critical system.

Furthermore, calculation of SSFMs is smoothed by averaging over many lattice positions (lattice averaged SSFMs, see Ref. [2]). An improved estimation of statistical errors of SSFMs is achieved by use of the bootstrap method [11,12,13], whereby the original set of events is resampled with replacement [2].

It is to be noted that, while individual $F_{2}(M)$ errors and confidence intervals can be estimated fairly well through the bootstrap, $F_{2}(M)$ errors for different $M$ are correlated, since the same data set is used in the calculation of all $F_{2}(M)$. Additional information about error correlations is contained in the full $F_{2}(M)$ correlation matrix, which can also be estimated through the bootstrap (see, for example, Ref. [11]). Furthermore, $\phi_{2}$ and its accompanying uncertainties should properly be determined not through a simple $\chi^{2}$-fit, but through a correlated fit. Unfortunately, such fits are plagued by instabilities [14]. We therefore resort to other methods in order to estimate $\phi_{2}$ uncertainties, such as individually fitting bootstrap samples to obtain a distribution of $\phi_{2}$ values and corresponding confidence intervals; however, present quoted $\phi_{2}$ uncertainties should be considered tentative.

A proton generating modification of the Critical Monte Carlo (CMC) code [6, 10] is used to simulate a system of critically correlated protons, which are mixed with a non-critical background to study the effects on the quality of intermittency analysis.

\section{Results}

\subsection{NA49 data analysis results}

An intermittency analysis performed on a number of different size NA49 collision systems at the maximum SPS energy [2] at mid-rapidity detected power-law fluctuations compatible with criticality in the "Si" $+\mathrm{Si}$ system, although with large statistical errors. Furthermore, no intermittency was detected in the " $\mathrm{C}$ " $+\mathrm{C}$ and $\mathrm{Pb}+\mathrm{Pb}$ systems at the same collision energy. Fig. 1 shows SSFMs $F_{2}(M)$ and $\Delta F_{2}(M)$ for the analyzed NA49 systems. For the "Si" $+\mathrm{Si}$ system, the intermittency index value was estimated, through the bootstrap, as $\phi_{2, B}=0.96_{-0.25}^{+0.38}($ stat. $) \pm 0.16$ (syst.) [2].

An interesting result emerges from simulating the NA49 "Si"+Si system, by mixing random (uncorrelated) simulated protons with critical protons produced via the aforementioned CMC code. The average ratio $\lambda$ of random noise to total proton event multiplicity is an adjustable parameter of such a simulation. Fig.2(a) shows the moments $F_{2}(M)$ of NA49 "Si" $+\mathrm{Si}$ data compared to a $\lambda=99 \%$ noise-contaminated CMC set, which approximates the former with fair accuracy. In

\footnotetext{
${ }^{1}$ This approximation is non-trivial to derive; the mixed term can be shown to be negligible through use of CMC simulation mixed with non-critical background.
} 
Fig.2(b), $\Delta F_{2}^{(e)}(M)$ is shown for the same CMC set, as well as the pure CMC; we notice that $\Delta F_{2}^{(e)}(M)$ of noisy CMC reproduces the slope (intermittency index $\phi_{2}$ ) of the pure CMC critical set, although their moments differ by orders of magnitude. Finally, Fig.2(c) shows the distribution of $\phi_{2}$ values across different bootstrap samples for the same noisy CMC set. The spread of $\phi_{2}$ values in the NA49 "Si"+Si system is reproduced and justified through mixing with background protons, whereas the median $\phi_{2}$ value approximates the theoretically expected intermittency index of the pure CMC. We conclude from noisy CMC simulations that indeed background is dominant in the NA49 data sets, and that the $\Delta F_{2}^{(e)}(M)$ approximation is valid in the case of dominant background.
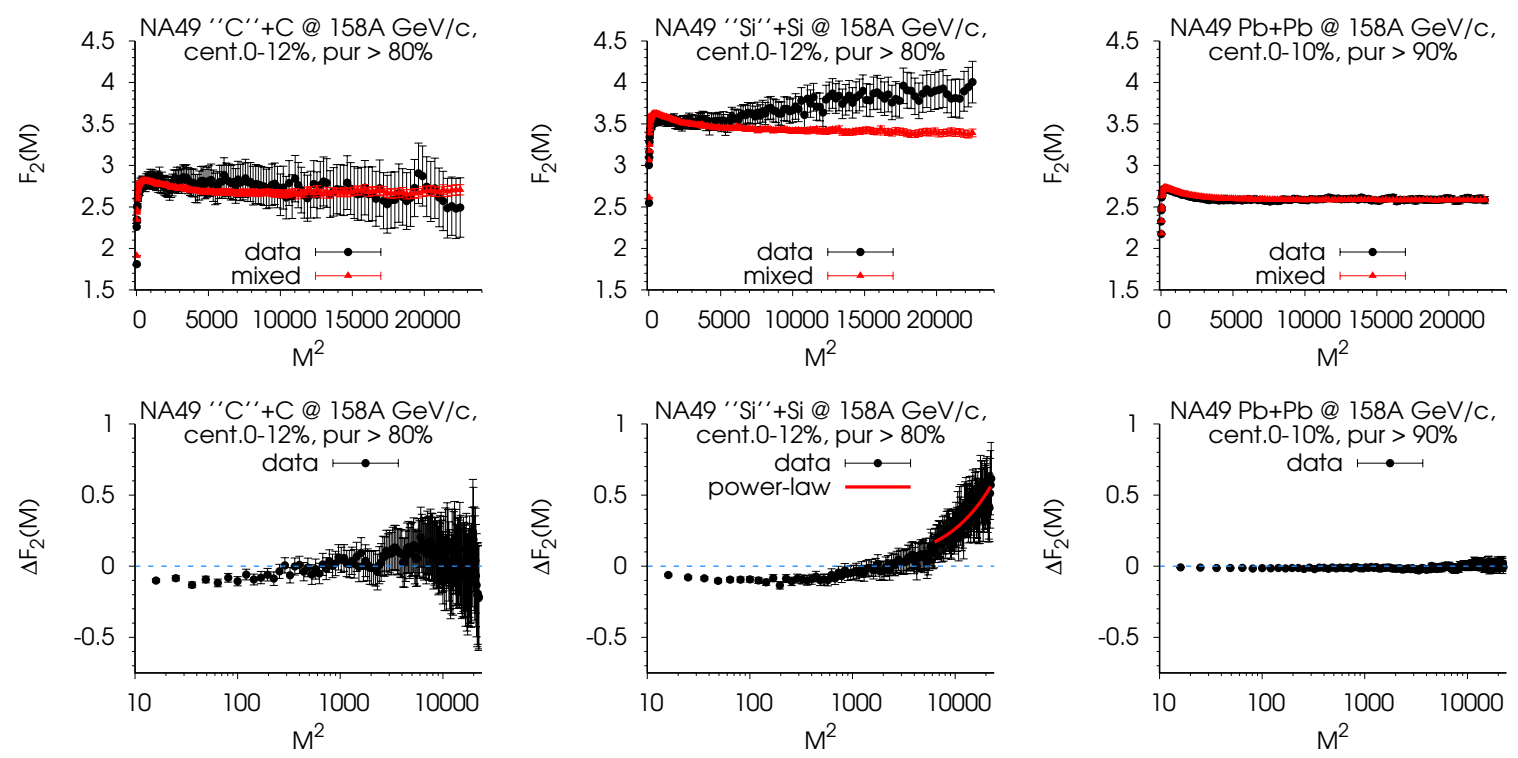

Figure 1: (top row) $F_{2}(M)$ of original (filled circles) and mixed events (filled triangles) for NA49 "C" $+\mathrm{C}$ (left), "Si" $+\mathrm{Si}$ (middle) and $\mathrm{Pb}+\mathrm{Pb}$ (right) collisions at $158 \mathrm{~A} \mathrm{GeV} / \mathrm{c}\left(\sqrt{s_{N N}}=17.3 \mathrm{GeV}\right)$. (bottom row) $\Delta F_{2}^{(e)}(M)$ for the corresponding systems. The " $\mathrm{Si}$ " $+\mathrm{Si}$ system (middle) is fitted with a power-law, $\Delta F_{2}^{(e)}\left(M ; \mathscr{C}, \phi_{2}\right)=e^{\mathscr{C}} \cdot\left(M^{2}\right)^{\phi_{2}}$, for $M^{2}>6000$.

\subsection{NA61/SHINE Be+Be preliminary data analysis}

The positive NA49 "Si" $+\mathrm{Si}$ result suggests we should look for intermittency in collisions of medium-sized nuclei recorded within the successor experiment NA61/SHINE. Intermittency analysis, if it is to be performed reliably, requires large event statistics (a minimum of $100 \mathrm{~K}$ events, ideally of the order of $1 \mathrm{M}$ events), reliable particle identification (candidate proton purity of at least $80-90 \%$ ), and a relatively high mean proton multiplicity density in mid-rapidity (at least 2 protons in the window of analysis, since we are looking for pair correlations). Subsequently, our two main candidate NA61 systems for study are ${ }^{7} \mathrm{Be}+{ }^{9} \mathrm{Be}$ and ${ }^{40} \mathrm{Ar}+{ }^{45} \mathrm{Sc}$ at $150 \mathrm{~A} \mathrm{GeV} / \mathrm{c}$.

Preliminary analysis of NA61/SHINE Be+Be at $150 \mathrm{~A} \mathrm{GeV/c}$ was presented in [15]. We analyzed a set of about $160 \mathrm{~K}, 12 \%$ most central events, with an average proton multiplicity of $1.48 \pm 0.74$ in the mid-rapidity range, excluding events with a zero proton multiplicity in this range. In Fig.3, intermittency analysis results are shown for NA61/SHINE Be+Be data. $F_{2}(M)$ for data and mixed events overlap; thus, $\Delta F_{2}(M)$ fluctuates around zero, and no intermittency effect is observed. 

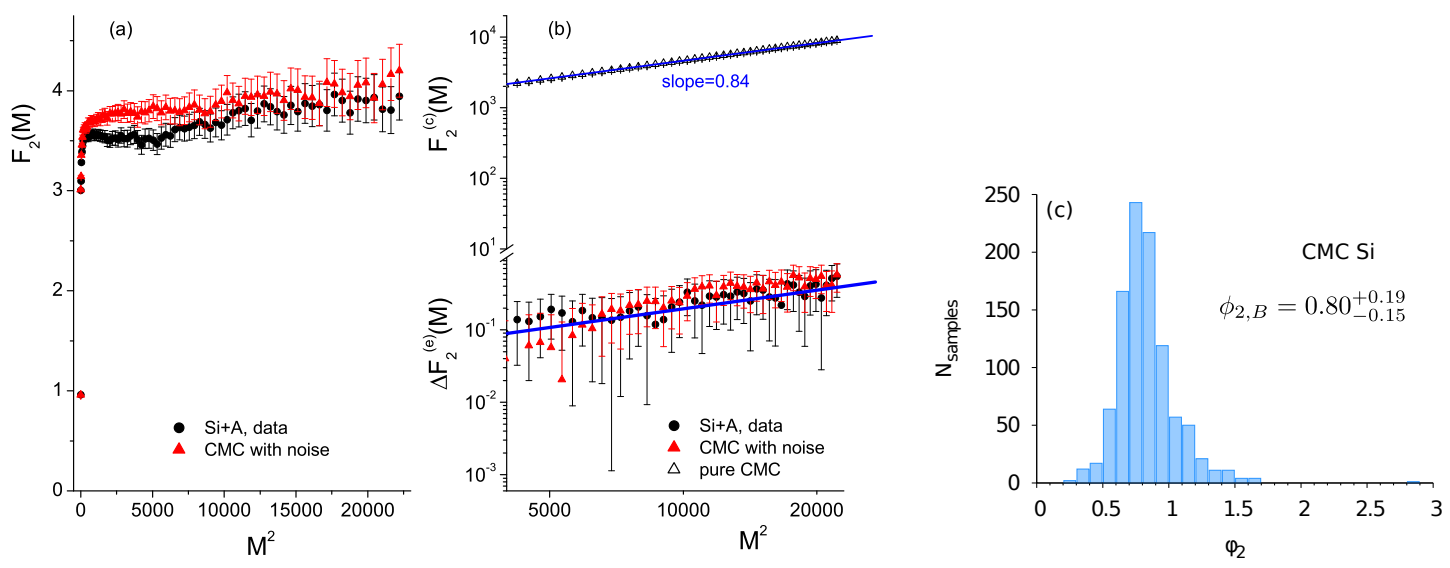

Figure 2: (a) $F_{2}(M)$ of NA49 "Si" $+\mathrm{Si}$ data (filled cirles) and noisy CMC simulated collisions (filled triangles) with $\lambda=99 \%$ background noise. (b) $F_{2}^{(c)}(M)$ for pure CMC system (open triangles), as well as the correlator $\Delta F_{2}^{(e)}(M)$ for noisy CMC. $\Delta F_{2}^{(e)}(M)$ is also shown for NA49 "Si"+Si data for comparison. The solid lines correspond to the theoretically expected slope $\phi_{2, c r}^{B}$. (c) $\phi_{2, B}$ bootstrap distribution for noisy "Si"+Si CMC. Asymmetric errors correspond to a $67 \%$ confidence interval.

Based on noisy Critical Monte Carlo simulation of the Be+Be system, we estimate an upper limit of the order of $0.3 \%$ for the fraction of critical protons in $\mathrm{Be}+\mathrm{Be}$ data to be consistent with the experimental observation.
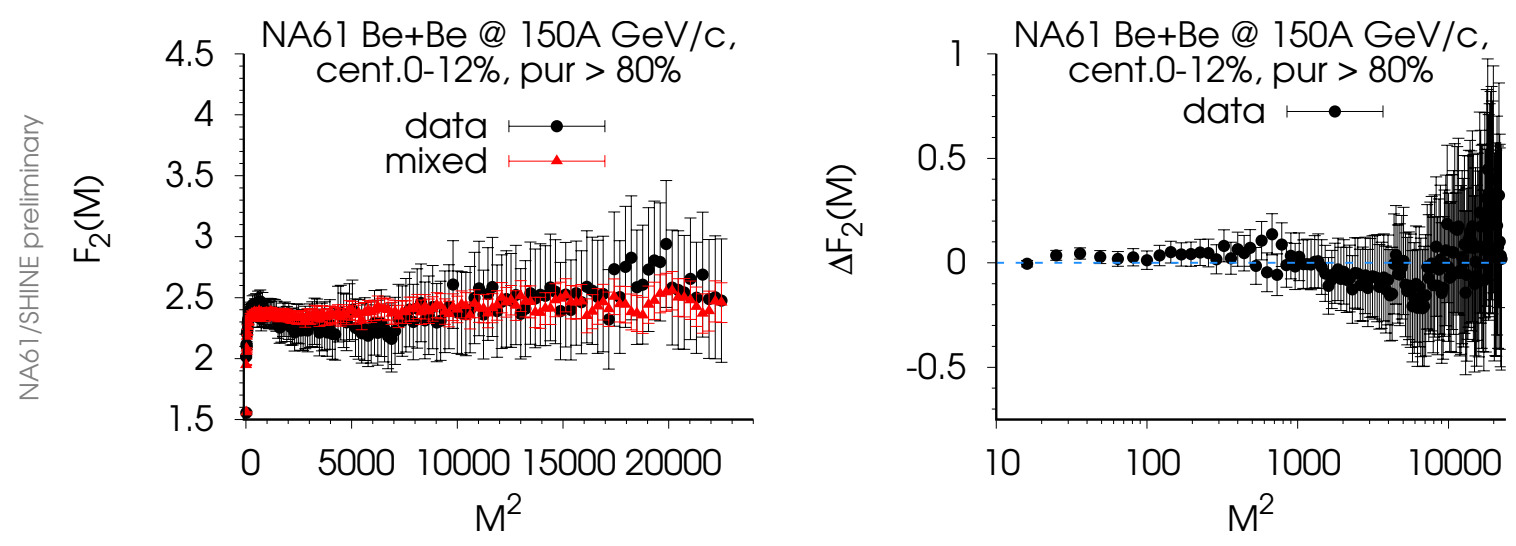

Figure 3: (left) $F_{2}(M)$ of protons in NA61/SHINE Be+Be collisions at $\sqrt{s_{N N}}=16.8 \mathrm{GeV}$, for data (black circles) and mixed events (red triangles). (right) $\Delta F_{2}^{(e)}(M)$ for the corresponding system.

\subsection{NA61/SHINE Ar+Sc intermittency analysis at $150 \mathrm{~A} \mathrm{GeV/c}$}

Following intermittency analysis on Be+Be, we now turn our focus to the NA61/SHINE ${ }^{40} \mathrm{Ar}+{ }^{45} \mathrm{Sc}$ system. We have analyzed 3 subsets of NA61/SHINE Ar+Sc events at $150 \mathrm{~A} \mathrm{GeV/c}$, corresponding to $0-5 \%, 5-10 \%$ and $10-15 \%$ most central collisions, determined by projectile spectator energy. Upon finding that intermittency analysis results depend considerably on the purity of selected protons, we have performed a scan on proton purity thresholds of $80 \%, 85 \%$, and $90 \%$. 
Table 1 summarizes the Ar+Sc statistics for all centralities and for $90 \%$ proton purity threshold. The average proton multiplicities are in all cases above the minimum required threshold of 2; however, event statistics is rather low, of the order of $150 \mathrm{~K}$ events. Consequently, SSFMs are subject to sizeable statistical uncertainties.

\begin{tabular}{rccc}
\hline Centrality & \#events & \multicolumn{2}{c}{$\langle p\rangle_{\left|p_{T}\right| \leq 1.5 \mathrm{GeV},\left|y_{C M}\right| \leq 0.75}$} \\
& & Non-empty & With empty \\
\hline $0-5 \%$ & 144,362 & $3.44 \pm 1.79$ & $3.30 \pm 1.89$ \\
$5-10 \%$ & 148,199 & $3.00 \pm 1.61$ & $2.79 \pm 1.73$ \\
$10-15 \%$ & 142,900 & $2.81 \pm 1.53$ & $2.58 \pm 1.66$ \\
\hline
\end{tabular}

Table 1: Event statistics and average proton multiplicity in the analysis window for the 3 analyzed centralities in NA61/SHINE Ar+Sc at 150A GeV/c. The 3rd column gives proton multiplicities after dropping empty (zero multiplicity) events; the 4th column, including zero multiplicity events.
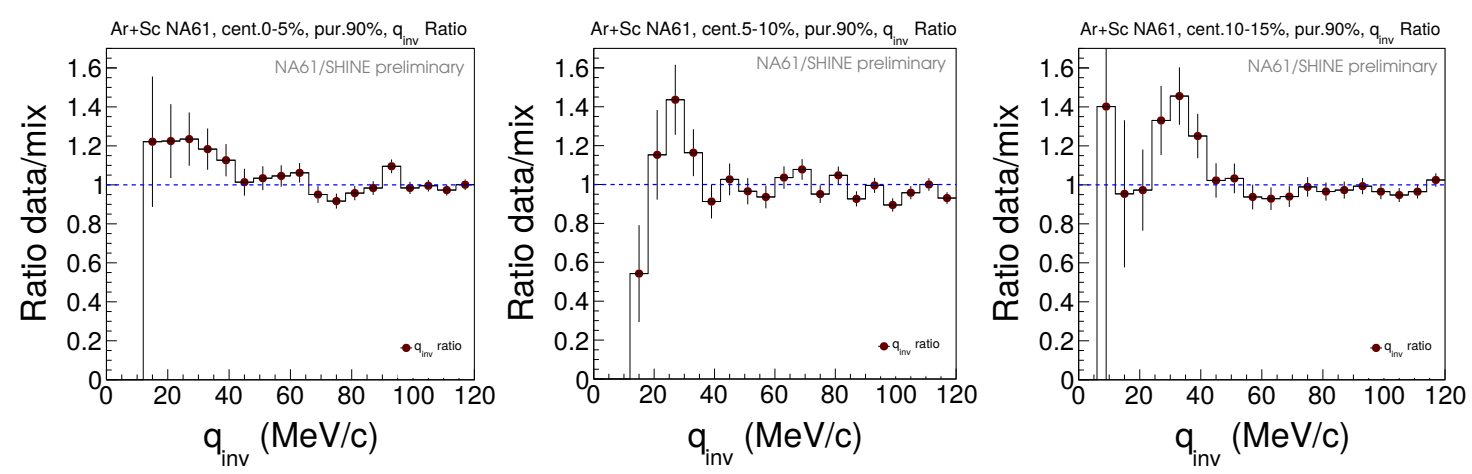

Figure 4: The ratio $P\left(q_{\text {inv }}\right)^{\text {data }} / P\left(q_{\text {inv }}\right)^{\text {mixed }}$ for a $90 \%$ minimum proton purity selection, for NA61 Ar+Sc collisions at $150 \mathrm{~A} \mathrm{GeV} / \mathrm{c}$, at $0-5 \%$ (left), $5-10 \%$ (middle) and $10-15 \%$ right centrality range.

Furthermore, it is necessary to apply additional quality cuts to selected protons before we proceed with an intermittency analysis. In particular, we must guard against the possibility of split tracks, i.e. sections of a track that are erroneously identified as a pair of distinct tracks, and could therefore compromise an analysis on correlations. To this end, we impose a minimum separation distance of accepted tracks in the detector. Additionally, we calculate, for original and mixed events, the distributions of invariant 4-momentum difference, $q_{i n v}$, of proton pairs:

$$
q_{i n v}\left(p_{i}, p_{j}\right) \equiv \frac{1}{2} \sqrt{-\left(p_{i}-p_{j}\right)^{2}}
$$

where $p_{i}$ is the 4-momentum of the $i$-th track. The ratio of distributions, $P\left(q_{\text {inv }}\right)^{\text {data }} / P\left(q_{\text {inv }}\right)^{\text {mixed }}$ for each centrality range is shown in Fig.4. The canonical form of the $q_{i n v}$ ratio is predicted [16] to have a peak around $20 \mathrm{MeV} / \mathrm{c}$ due to strong interactions and to be suppressed for lower $q_{\text {inv }}$ due to Fermi-Dirac effects and Coulomb repulsion; thus, peaks at low $q_{i n v}$ indicate possible split track contamination that must be removed. Based on the observed $q_{i n v}$ ratios for $\mathrm{Ar}+\mathrm{Sc}$, we impose a universal cutoff of $q_{i n v}>7 \mathrm{MeV} / \mathrm{c}$ to all sets before analysis.

Having made a proton selection, we can then perform a first check for the presence of intermittency in transverse momentum space by defining the transverse momentum difference, $\Delta p_{T}$, of proton pairs, in direct correspondence to $q_{i n v}$ : 

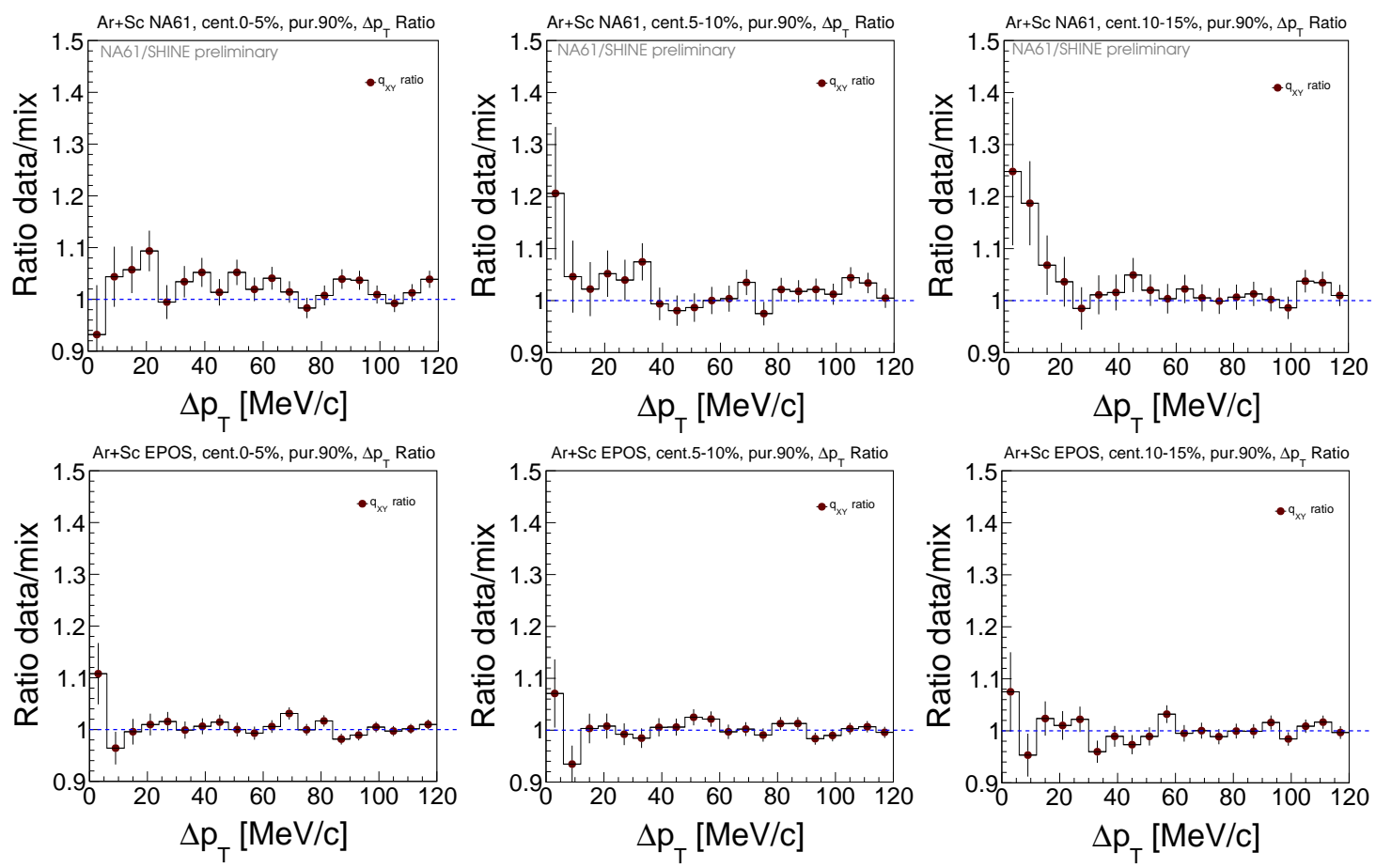

Figure 5: (top row) The ratio $P\left(\Delta p_{T}\right)^{\text {data }} / P\left(\Delta p_{T}\right)^{\text {mixed }}$ for a $90 \%$ minimum proton purity selection, for $\mathrm{NA} 61 \mathrm{Ar}+\mathrm{Sc}$ collisions at $150 \mathrm{~A} \mathrm{GeV} / \mathrm{c}$, at $0-5 \%$ (left), 5-10\% (middle) and 10-15\% right centrality range. (bottom row) corresponding $\Delta p_{T}$ ratios for EPOS [17] simulated Ar+Sc collisions of the same centrality.

$$
\Delta p_{T} \equiv \frac{1}{2} \sqrt{\left(p_{X_{1}}-p_{X_{2}}\right)^{2}+\left(p_{Y_{1}}-p_{Y_{2}}\right)^{2}}
$$

Fig.5 shows the ratio $P\left(\Delta p_{T}\right)^{\text {data }} / P\left(\Delta p_{T}\right)^{\text {mixed }}$ for the 3 centralities in $\mathrm{Ar}+\mathrm{Sc}$, in comparison with the corresponding ratios for a simulation of the Ar+Sc system via the EPOS event generator [17]. We notice an indication of power-law scaling for $\Delta p_{T} \rightarrow 0$ for middle-central (5-10\% \& 1015\%) NA61/SHINE Ar+Sc collisions; no clear power-law structure is visible in the corresponding EPOS spectra.

Finally, we proceed to the intermittency analysis proper; we calculate the SSFMs $F_{2}(M)$ and $\Delta F_{2}(M)$ for the $3 \times 3$ centrality and proton purity selections. Fig. 6 shows the results for $90 \%$ proton purity selection, for the NA61/SHINE data sets. In agreement with the preliminary $\Delta p_{T}$ probe, we observe a significant separation of $F_{2}(M)$ of data from those of mixed events for the 10$15 \%$ centrality case; a weaker effect is seen for $5-10 \%$ most central collisions, while central (0-5\%) collisions show a total overlap of moments (no intermittency effect). At present, the uncertainties of $\Delta F_{2}(M)$, as well as the fact that they are correlated, do not permit a safe estimation of the quality of power-law or the calculation of confidence intervals for $\phi_{2}$.

By contrast, Fig.7 shows the corresponding $F_{2}(M)$ and $\Delta F_{2}(M)$ for the EPOS simulated collisions. We observe that EPOS fails to reproduce the scaling of $\Delta F_{2}(M)$ for mid-central collisions: there is always significant overlap of data and mixed event moments; the power-law fits (red solid lines) are drawn merely to guide the eye, as the fits fail due to the prevalence of negative $\Delta F_{2}(M)$ values. 

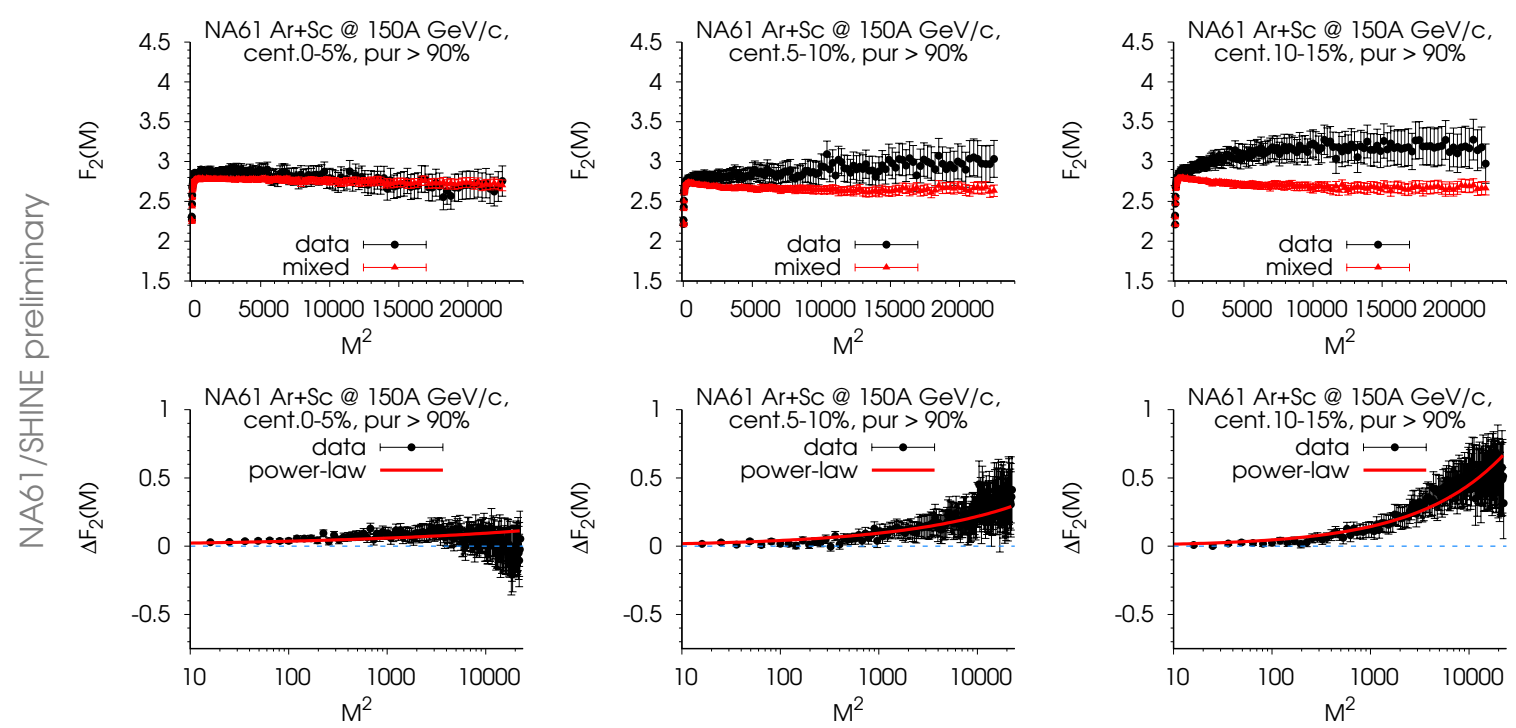

Figure 6: (top row) $F_{2}(M)$ of original (filled circles) and mixed events (filled triangles) for NA61 Ar+Sc collisions at $0-5 \%$ (left), $5-10 \%$ (middle) and $10-15 \%$ (right) centrality at $150 A \mathrm{GeV} / \mathrm{c}\left(\sqrt{s_{N N}}=16.8 \mathrm{GeV}\right)$. (bottom row) $\Delta F_{2}^{(e)}(M)$ for the corresponding systems. The solid curves are drawn to guide the eye and correspond to power-law scaling functions, $\Delta F_{2}^{(e)}\left(M ; \mathscr{C}, \phi_{2}\right)=e^{\mathscr{C}} \cdot\left(M^{2}\right)^{\phi_{2}}$, with parameters: (left) $\phi_{2}=0.21, \mathscr{C}=-4.27$; (middle) $\phi_{2}=0.36, \mathscr{C}=-4.84$; (right) $\phi_{2}=0.49, \mathscr{C}=-5.4$.

Figs.8,9 summarize the scan in centrality and proton purity for NA61/SHINE Ar+Sc data. In general, we see a tendency for increased $\Delta F_{2}(M)$ scaling as we increase peripherality and proton purity.

\section{Summary and conclusions}

The study of self-similar (power-law) fluctuations of the proton density in transverse momentum space through intermittency analysis provides us with a promising set of observables for the detection of the QCD critical point. The intermittency analysis performed on NA49 "Si"+Si data at the maximum SPS energy already indicates the presence of a critical proton component of the order of $1 \%$, with an estimated intermittency index value of $\phi_{2, B}=0.96_{-0.25}^{+0.38}$, overlapping with the critical QCD prediction, whereas no intermittency is observed in either the smaller " $\mathrm{C}$ " $+\mathrm{C}$ or the larger $\mathrm{Pb}+\mathrm{Pb}$ system at the same collision energy. The preliminary analysis of the NA61/SHINE central $\mathrm{Be}+\mathrm{Be}$ system at $150 \mathrm{~A} \mathrm{GeV/c}$ consistently provides a negative result.

Our preliminary study of NA61/SHINE central and middle-central $\mathrm{Ar}+\mathrm{Sc}$ collisions at $150 \mathrm{~A} \mathrm{GeV/c}$ offers the first indication of a non-trivial intermittency effect. We see an indication of power-law scaling in the SSFMs $\Delta F_{2}(M)$ of Ar+Sc collisions; the quality of the effect seems to increase for less central collisions, as well as for increased proton purity thresholds, up to $90 \%$ purity. EPOS simulations of the corresponding systems fail to reproduce the effect. However, due to the magnitude of SSFMs uncertainties, and the fact that $F_{2}(M)$ values for distinct $M$ are correlated, the quality of $\Delta F_{2}(M)$ power-law scaling remains still to be established, and an estimation of $\phi_{2}$ confidence intervals is still pending. 

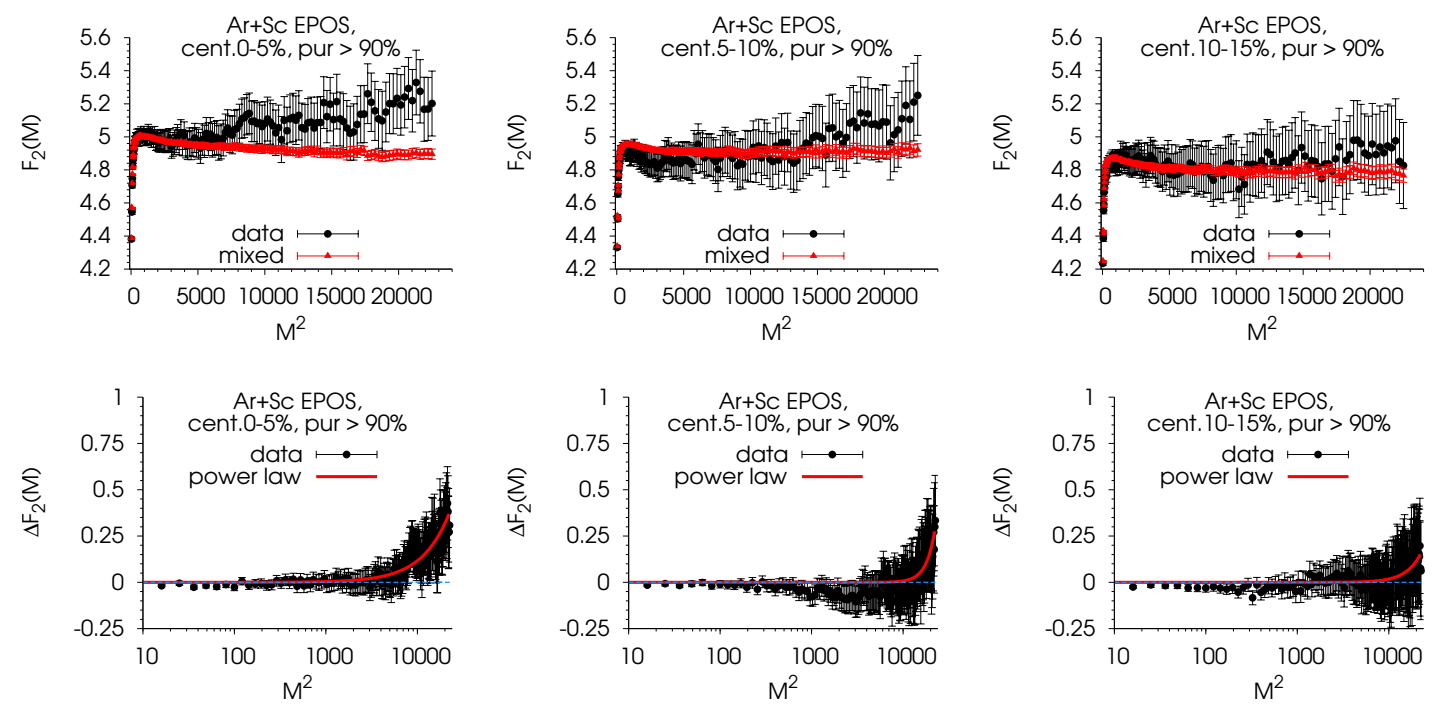

Figure 7: (top row) $F_{2}(M)$ of original (filled circles) and mixed events (filled triangles) for EPOS Ar+Sc collisions at $0-5 \%$ (left), $5-10 \%$ (middle) and $10-15 \%$ (right) centrality at $150 \mathrm{~A} \mathrm{GeV} / \mathrm{c}\left(\sqrt{s_{N N}}=16.8 \mathrm{GeV}\right)$. (bottom row) $\Delta F_{2}^{(e)}(M)$ for the corresponding systems. The solid curves are drawn to guide the eye and correspond to power-law scaling functions, $\Delta F_{2}^{(e)}\left(M ; \mathscr{C}, \phi_{2}\right)=e^{\mathscr{C}} \cdot\left(M^{2}\right)^{\phi_{2}}$.

A continued, final analysis of the total available statistics of NA61/SHINE Ar+Sc data, and its extension to other system sizes (Xe+La) and energies of the NA61/SHINE program will hopefully lead to an accurate determination of the critical point location.

Acknowledgments: This work was supported by the National Science Centre, Poland (grant no. 2014/14/E/ST2/00018).

\section{References}

[1] K. Fukushima and T. Hatsuda, Rep. Prog. Phys. 74, 014001 (2011).

[2] T. Anticic et al, Eur. Phys. J. C 75: 587 (2015).

[3] C. Alt et al., Phys. Rev. C 75, 064904 (2007); Phys. Rev. C 78, 034914 (2008).

[4] L. Adamczyk et al., Phys. Rev. Lett. 112, 032302 (2014).

[5] T. Anticic et al., Phys. Rev. C 70, 034902 (2004); Phys. Rev. C 79, 044904 (2009).

[6] N. G. Antoniou, Y. F. Contoyiannis, F. K. Diakonos, A. I. Karanikas and C. N. Ktorides, Nucl. Phys. A 693, 799 (2001).

[7] N. G. Antoniou, Y. F. Contoyiannis, F. K. Diakonos and G. Mavromanolakis, Nucl. Phys. A 761, 149 (2005).

[8] Y. Hatta and M. A. Stephanov, Phys. Rev. Lett. 91, 102003 (2003).

[9] T. Vicsek, Fractal Growth Phenomena (World Scientific, Singapore, 1989). ISBN 9971-50-830-3

[10] N. G. Antoniou, F. K. Diakonos, A. S. Kapoyannis and K. S. Kousouris, Phys. Rev. Lett. 97, (2006) 032002 . 
[11] W. J. Metzger, "Estimating the Uncertainties of Factorial Moments", HEN-455 (2004).

[12] B. Efron, Ann. Stat. 7, 1 (1979).

[13] T. Hesterberg et al., Bootstrap Method and Permutation Tests (W. H. Freeman \& Co., USA, 2003). ISBN-10:0716757265

[14] C. Michael, Phys. Rev. D 49, 2616-2619 (1994).

[15] N. Davis, for the NA61/SHINE Collaboration, PoS (CPOD2017) 054.

[16] S. E. Koonin, Phys. Let. B 70, 43-47 (1977).

[17] K. Werner, F. Liu, T. Pierog, Phys. Rev. C 74, 044902 (2006). 

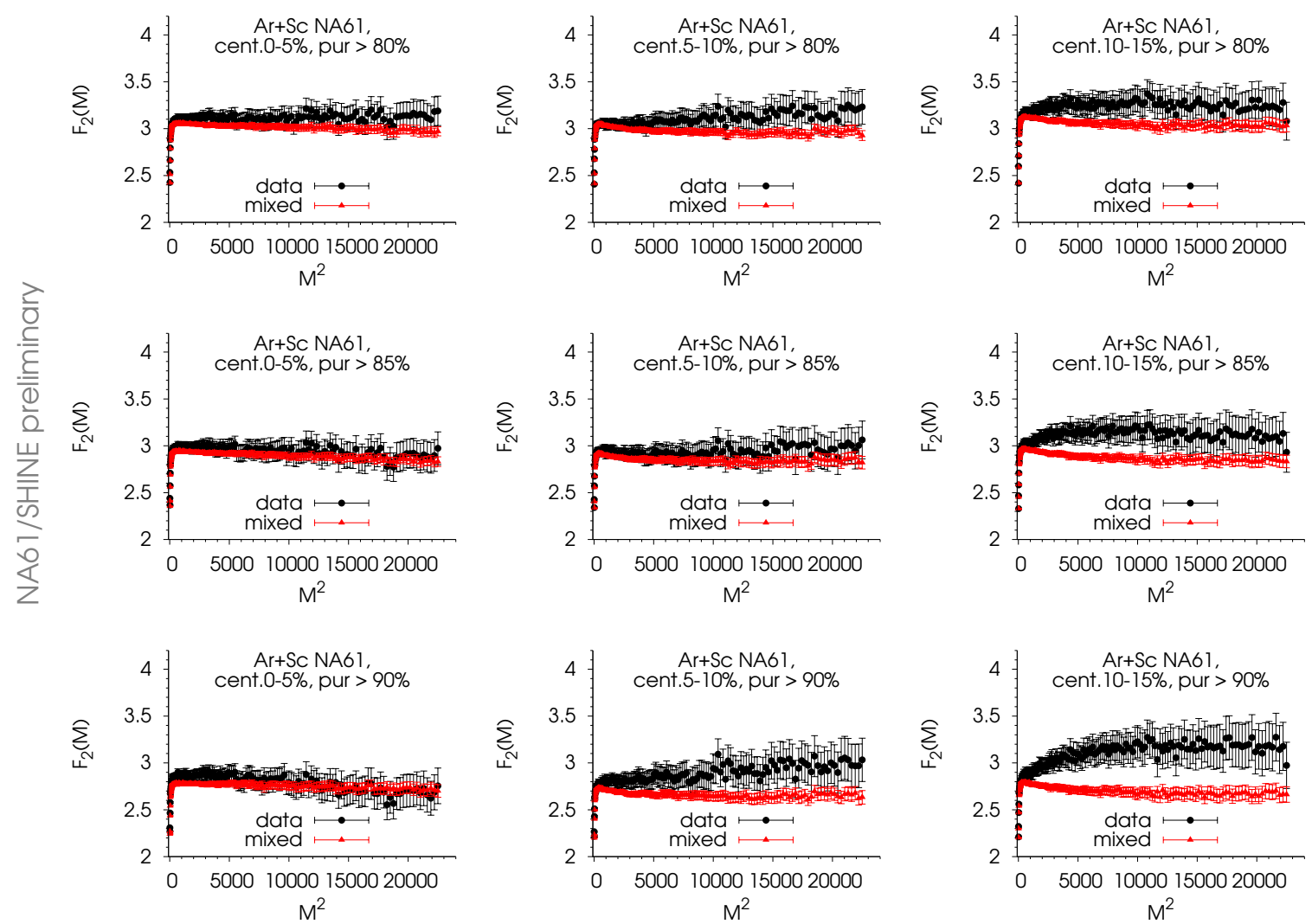

Figure 8: $F_{2}(M)$ of original (filled circles) and mixed events (filled triangles) for NA61 Ar+Sc collisions at $0-5 \%$ (left), $5-10 \%$ (middle) and 10-15\% (right) centrality at $150 \mathrm{~A} \mathrm{GeV/c}$, for $80 \%$ (top), $85 \%$ (center), and $90 \%$ (bottom) proton purity thresholds. 

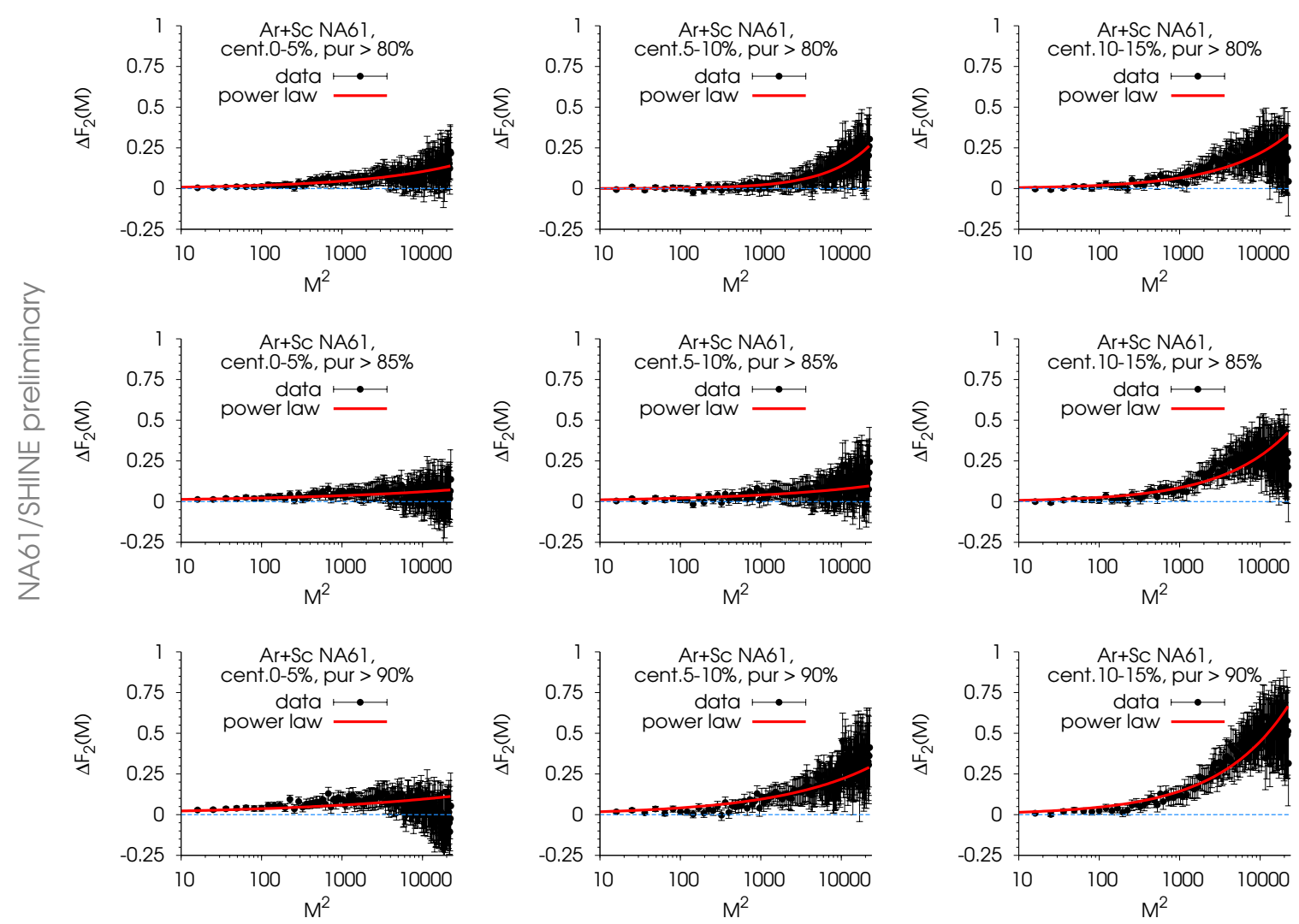

Figure 9: $\Delta F_{2}(M)$ of original (filled circles) and mixed events (filled triangles) for NA61 Ar+Sc collisions at $0-5 \%$ (left), $5-10 \%$ (middle) and $10-15 \%$ (right) centrality at $150 \mathrm{~A} \mathrm{GeV/c}$, for $80 \%$ (top), $85 \%$ (center), and $90 \%$ (bottom) proton purity thresholds. 